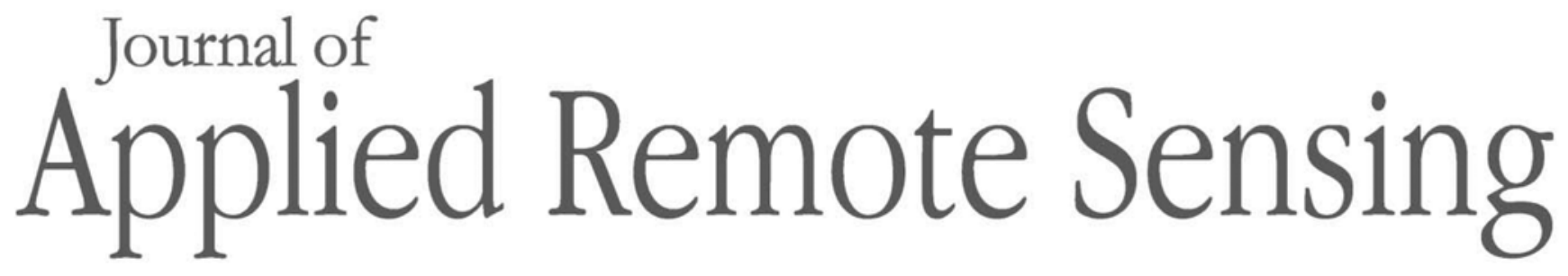

RemoteSensing.SPIEDigitalLibrary.org

\title{
Learning vector quantization neural network for surface water extraction from Landsat OLI images
}

Deepakrishna Somasundaram

Fangfang Zhang

Shenglei Wang

Huping Ye

Zongke Zhang

Bing Zhang 


\title{
Learning vector quantization neural network for surface water extraction from Landsat OLI images
}

\author{
Deepakrishna Somasundaram, ${ }^{\text {a,b }}$ Fangfang Zhang, ${ }^{\text {a }}$ Shenglei Wang, ${ }^{\text {a,c }}$ \\ Huping Ye, ${ }^{\mathrm{d}}$ Zongke Zhang, ${ }^{\text {,f }}$ and Bing Zhang, ${ }^{\mathrm{a}, \mathrm{b}, *}$ \\ ${ }^{a}$ Chinese Academy of Sciences, Institute of Remote Sensing and Digital Earth, \\ Key Laboratory of Digital Earth Science, Beijing, China \\ ${ }^{b}$ University of Chinese Academy of Sciences, Beijing, China \\ ${ }^{c}$ Peking University, Institute of Remote Sensing and Geographic Information System, \\ Beijing, China \\ ${ }^{\mathrm{d}}$ Chinese Academy of Sciences, Institute of Geographic Sciences and \\ Natural Resources Research, State Key Laboratory of Resources and \\ Environmental Information System, Beijing, China \\ ${ }^{\mathrm{e}}$ Chinese Academy of Sciences, China-Sri Lanka Joint Research and \\ Demonstration Center for Water Technology, Beijing, China \\ ${ }^{\mathrm{f}}$ Chinese Academy of Sciences, China-Sri Lanka Joint Center for Education and Research, \\ Guangzhou, China
}

\begin{abstract}
There is a growing concern over surface water dynamics due to an increased understanding of water availability and management with current climate trends. Remote sensing has now become an effective means of water extraction due to the availability of an enormous amount of data with diverse spatial, spectral, and temporal resolutions. However, water extraction from optical remote sensing data is associated with several major difficulties, such as the applicability of the extraction method over large areas and complex environments; shadow contamination from clouds, buildings, and mountains; and disclosure of shadowed water and exclusion of floating and submerged plants. To address these difficulties, a learning vector quantization (LVQ) neural network-based method was proposed and implemented to extract water using Landsat 8 imageries. This method is capable of separating water from clouds, build-up areas, shadows, and shadowed water by the ideal input of bands 1 to 7 and normalized difference vegetation index. This model learns water across Sri Lanka. Eight OLI scenes were tested, and the performance was compared with five widely used machine learning algorithms: support vector machine, K-nearest neighbor, discriminant analysis, combination of modified normalized difference water index and modified fuzzy clustering method, and K-means clustering methods. This method performed the best, achieving overall accuracies and the kappa coefficients between $97.8 \%$ and $99.7 \%$ and between 0.96 and 0.99 , respectively. Results have demonstrated robustness, consistency, and preciseness in various dark surfaces, noisiest water environments, and highly water scarce scenes. LVQ revealed a good generalizing ability to detect all types of water with less amount of training samples. This method can be easily adaptable for other sensors and global water to support water resource studies. () The Authors. Published by SPIE under a Creative Commons Attribution 4.0 Unported License. Distribution or reproduction of this work in whole or in part requires full attribution of the original publication, including its DOI. [DOI: 10.1117/1.JRS.14.032605]
\end{abstract}

Keywords: water extraction; water body mapping; learning vector quantization; neural network; machine learning; Landsat 8.

Paper 190651SS received Aug. 25, 2019; accepted for publication Dec. 19, 2019; published online Jan. 14, 2020.

\section{Introduction}

Remote sensing is an efficient technique for monitoring and extracting surface water ${ }^{1,2}$ and has been extensively utilized and has become a primary source for numerous studies relevant to

*Address all Correspondence to Bing Zhang, E-mail: zb@radi.ac.cn 
water as it provides fine spatial, multispectral, lower cost, and greater temporal views of surface water over larger areas, compared to the ground measurements. ${ }^{3}$ Many methods have been developed to extract water features from optical remote sensing data, such as thresholding and machine learning. ${ }^{4}$ Threshold-based water indices are broadly adapted to identify surface water due to simplification, whereas these indices are relying on a locally varying optimal threshold, which hinders the universal applicability on a broadscale. ${ }^{1}$ Due to the availability of the high amount of data and large-scale applications, a considerable number of studies have investigated automated processes of feature recognition and reform. The machine learning methods extremely absorb data from training samples to optimally separate water from nonwater in automated manner, including artificial neural network (ANN), ${ }^{2}$ random forest,${ }^{5}$ decision trees, ${ }^{6}$ isocluster, ${ }^{7}$ K-nearest neighbor $(\mathrm{KNN}),{ }^{8}$ discriminant analysis (DA), ${ }^{2}$ fuzzy clustering, ${ }^{9}$ $\mathrm{K}$-means clustering, ${ }^{10}$ and support vector machine (SVM). ${ }^{3}$ Studies have demonstrated that the primary benefit of these models is the higher classification accuracy in a more complex system. ${ }^{11,12}$ During the last three decades, ANNs have undergone a rapid improvement in most research areas ${ }^{13,14}$ due to their learning ability, relative noise tolerance, unreliableness on data distribution, and great parallel structure, including target detection, image segmentation and image classification, ${ }^{15-17}$ climate and environmental transformations, ${ }^{18}$ and remote sensing water mapping. ${ }^{19-21}$

Learning vector quantization (LVQ) is an adaptive heuristic method for data classification due to simplicity, faster convergence, stability, better generalization, flexibility, robustness, ${ }^{22-25}$ and more computational efficiency than other ANN techniques. ${ }^{26,27}$ The complexity of remote sensing images has been aggravated due to the advancement of sensors and the existence of various environmental noises. Such inevitable circumstances can be solved by learning further in detail and deliberate much information from many bands. ${ }^{28}$ In addition to the spectral characteristics, shape, textural information, and the spatial dependence between the labels of neighboring pixels also iteratively learned by LVQ to maximize the classification accuracy. It applies unsupervised techniques for data clustering while using a supervised training method. ${ }^{25}$ This has been commonly used in remote sensing applications involving image classification, ${ }^{29,30}$ land use classification, ${ }^{31,32}$ and water extraction with contrasting results. ${ }^{4}$ Although there are several studies in the field of remote sensing, the application of the LVQ in surface water extraction has not been clearly presented in the literature. Most existing applications of LVQ only used a few bands of the sensors and were conducted on a small scale. ${ }^{4,32}$ This has motivated us to employ the LVQ neural network in the process of water extraction and demonstrate its ability, applicability, and consistency in a country scale against various water methods using maximal band information.

Though numerous methods have been proposed to extract surface water from optical data, each has its own accuracy and functional difficulties due to the obstacles. One prominent obstacle for accurate water extraction is low reflectance surfaces, which have similar reflectance with water, such as terrain and cloud shadows and dark build-up. ${ }^{1,33}$ Secondly, shadowed water and floating plants, especially in the study region, where predominantly cloudy with higher aquatic plant diversity. ${ }^{34}$ Therefore, water mapping in Sri Lanka is challenging, where the decision boundary is much more complex. Those water bodies are not only small and numerous but also diverse in water condition, such as size, shape, depth, altitude, temperature, vegetation cover, and surroundings, moreover, associated ground details with heterogeneity in space. Prevailing climatic dynamics also necessitate the critical task of continuous monitoring in a complex environment for decision-making. Hence, it is necessary to explore the most appropriate and useful method for the purpose of water mapping throughout Sri Lanka. We attempt to address the aforementioned problems by proposing an LVQ-based method to increase the accuracy of water extraction under different confused situations. The objective of this study is to develop an LVQ-based model that can reliably extract water with high accuracy under various obstructions and environmental noises. The robustness of this proposed method is comprehensively evaluated and compared with other five widely used machine learning algorithms. 


\section{Study Area and Data}

\subsection{Study Areas}

Sri Lanka is located in the tropics, which has a higher number of small- and medium-sized surface water resources. The study area is situated between $5^{\circ} 55^{\prime} \mathrm{N}$ to $9^{\circ} 51^{\prime} \mathrm{N}$ latitude and $79^{\circ} 41^{\prime} \mathrm{E}$ to $81^{\circ} 53^{\prime} \mathrm{E}$ longitude and eight typical test sites are shown in Fig. 1. These test sites represent a wide range of water bodies with various types (i.e., ponds, lakes, reservoirs, streams, rivers, ocean), sizes, and depth, and their background environment include diverse land cover types, such as build-up areas, forest, paddy fields with flat, hilly, and mountainous terrains. A range of environmental noise to evaluate the performance of the proposed model and a brief description of test sites is presented in Table 1.

\subsection{Data}

For this study, four Landsat 8 OLI images (15 March 2016: path 141, rows 55, 56; 13 January 2017: path 141, rows 55 and 13 September 2018: path 141, rows 54) were collected from the U.S. Geological Survey (USGS). ${ }^{35}$ Images were Level 1 terrain-corrected (L1T) and pregeoreferenced using the WGS84 datum. Sentinel-2 images belonging to five tiles at 10-m resolution were used as reference data and were collected from the European Space Agency (ESA). ${ }^{36}$ The corresponding metadata information and a brief description of each test scene are summarized in Table 1.

\section{Methods}

The overall methodology adopted in this study for the identification and extraction of water bodies is shown in Fig. 2. This LVQ-based method consists of five stages: (1) applying

(a)

(b)

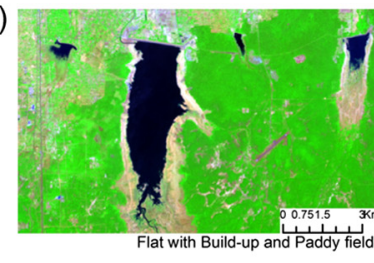

(c)

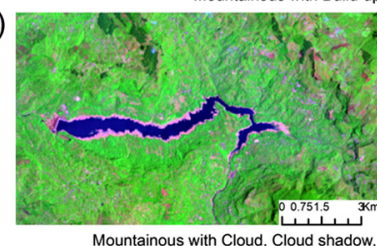

(d)
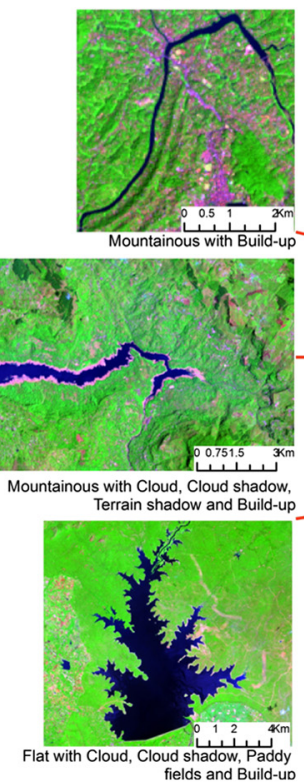
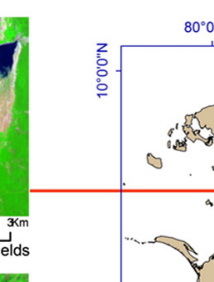

$0^{\circ} 0^{\prime} 0^{\prime \prime} E$

$81^{\circ} 0^{\prime} 0 \mathrm{~B}$
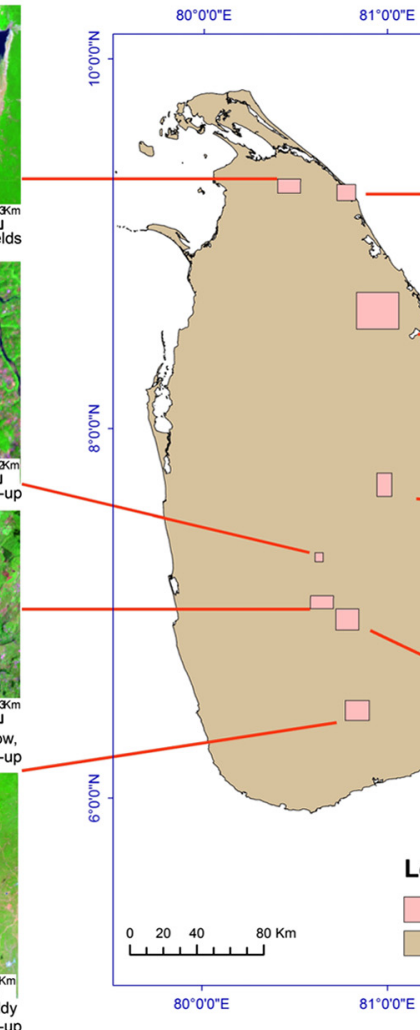

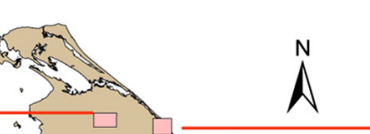

Fig. 1 Locations of the selected study area and test sites in Sri Lanka represent different conditions of water in the presence of various environmental noises (Landsat OLI band composite: R: band 7; G: band 5; B: band 2). (e)

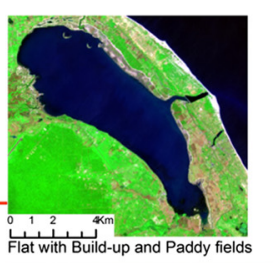

(f)

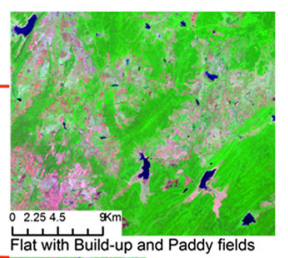

g)

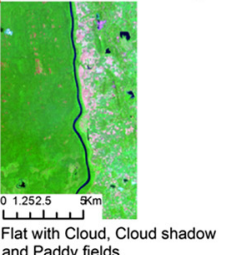

(h)

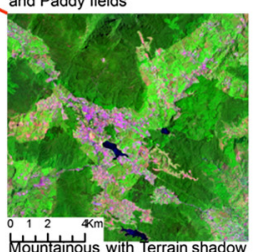

Study Area 
Somasundaram et al.: Learning vector quantization neural network for surface water extraction...

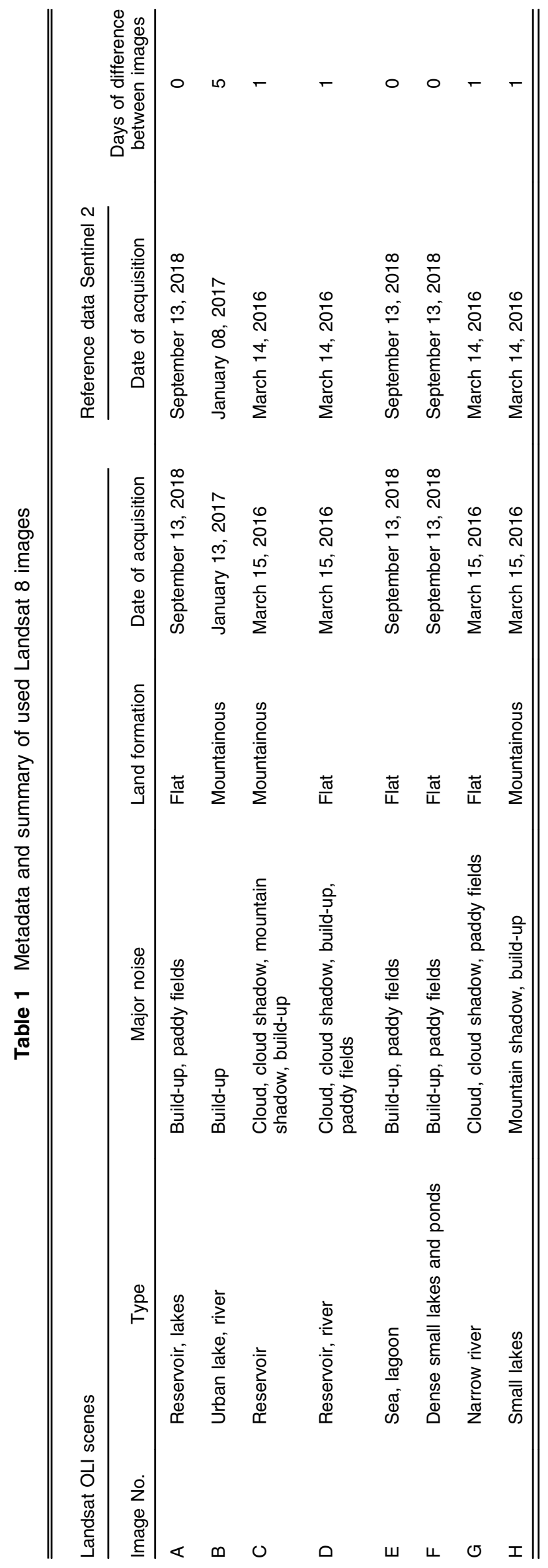




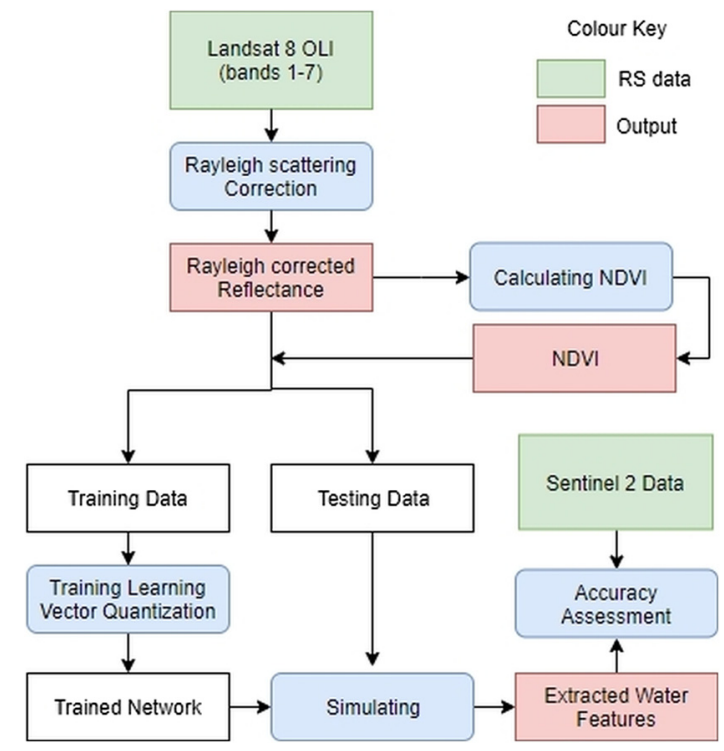

Fig. 2 Flowchart of adapted methodology in this study.

Rayleigh correction; (2) selecting training samples; (3) designing and training network; (4) simulating; and (5) assessing the accuracy. More detailed descriptions of each step are described in the following sections.

\subsection{Image Preprocessing}

Radiometric calibration and atmospheric corrections are prerequisites for raw Landsat imagery to obtain identical and high-quality experimental data. ${ }^{37}$ Due to the challenges associated with atmospheric correction over inland and coastal water, Rayleigh-corrected reflectance has been widely used in water applications with consistency. ${ }^{38}$ Images were processed and Rayleighcorrected reflectance were derived using the Atmospheric Correction for OLI lite tool (acolite_win_2014).

\subsection{Training Sample Selection}

Uniformly distributed training samples are efficient to train ANN. ${ }^{2}$ Training samples are selected across the study area from four OLI images except test areas. A total of 3765 water and 2685 nonwater pixels were selected in order to examine the efficiency of LVQ and accelerate the training process. Selection and distribution of samples are done by experience following a series of experiments. Pixels that are recognized as true water were exclusively selected for the training process to ensure the accuracy and quality of the network. All samples are manually labeled using region of interest polygons as water and nonwater using ENVI 5.3 software.

\subsection{Training $L V Q$ Network}

A total of eight layers, including OLI bands from one to seven and normalized difference vegetation index (NDVI), were included in the network together with the per-pixel class label. The LVQ network was constructed by the newlvq function in the neural network toolbox provided by MATLAB R2014b. Parameters were selected according to a rigorous examination of a series of experiments with higher efficiency and minimum computational error. ${ }^{39}$ Learning rates of 0.01 , 0.001 , and 0.0001 have experimented; both learning rates 0.01 and 0.001 were excluded as they converged at a lower number of epochs and 0.0001 converges around epochs 200 . The experiment result showed how training and classification accuracies evolve with the number of epochs and time (Fig. 3). We set the number of iterations and neurons as 200 and 50, respectively, in concern of an optimization strategy. 


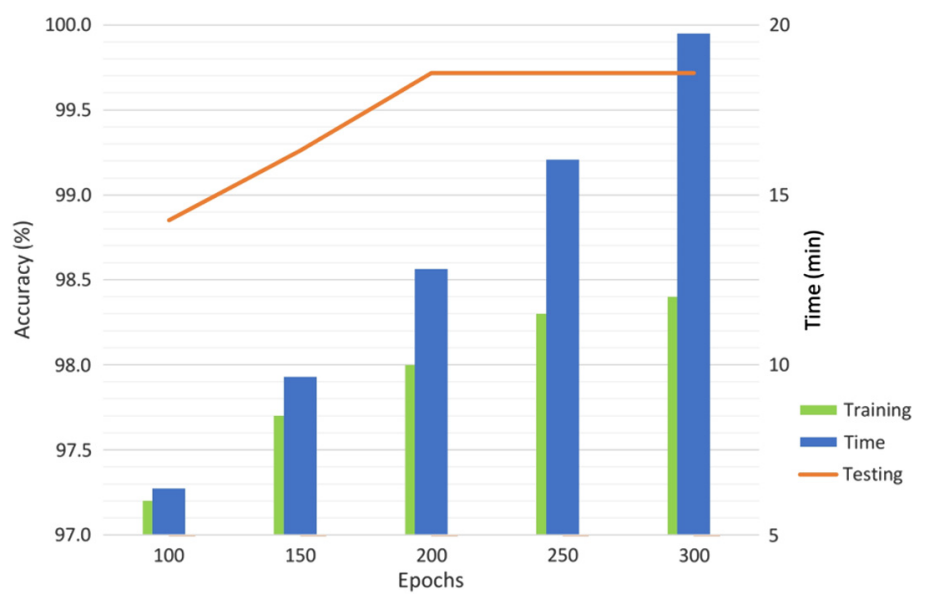

Fig. 3 Investigation of accuracy, time on the number of epochs at a learning rate of 0.0001 .

\subsection{Simulation}

According to the trained LVQ, each pixel was classified into two categories: water or nonwater. The sim function in the MATLAB neural network toolbox was applied to simulate the trained network and a binary image was obtained as a result with different color labels.

\subsection{Accuracy Assessment}

The performance evaluation for entire scenes is conducted in a quantitative manner and visual comparison. All the eight Landsat image scenes are verified with the corresponding highresolution Sentinel-2 images and a confusion matrix is calculated. All water pixels and an equal amount of nonwater pixels are used to assess the accuracy. Performance evaluation was done by four accuracy measures, including overall accuracy, kappa coefficient, producer's accuracy, and user's accuracy, which were defined by following standard equations: ${ }^{40}$

$$
\text { Overall accuracy }=\frac{(\mathrm{TP}+\mathrm{TN})}{\mathrm{N}} \times 100
$$

$$
\begin{gathered}
\text { Kappa coefficient }=\frac{N(\mathrm{TP}+\mathrm{TN})-[(\mathrm{TP}+\mathrm{FP})(\mathrm{TP}+\mathrm{FN})+(\mathrm{FN}+\mathrm{TN})(\mathrm{FP}+\mathrm{TN})]}{N^{2}-[(\mathrm{TP}+\mathrm{FP})(\mathrm{TP}+\mathrm{FN})+(\mathrm{FN}+\mathrm{TN})(\mathrm{FP}+\mathrm{TN})]}, \\
\text { Producer's accuracy }=\frac{\mathrm{TP}}{(\mathrm{TP}+\mathrm{FN})}, \\
\text { User's accuracy }=\frac{\mathrm{TP}}{(\mathrm{TP}+\mathrm{FP})},
\end{gathered}
$$

where TP is true positive: the number of detected water pixels, TN is true negative: the number of detected nonwater pixels, FP is false positive: the number of falsely detected water pixels, FN is false negative: the number of undetected water pixels, and $N$ is the total number of pixels used in accuracy assessment.

\section{Results}

\subsection{Water Extraction Result and Quantitative Assessment}

With the water extraction process proposed, results in binary maps in all test sites are shown in Fig. 4. A significant amount of clouds, cloud shadows, terrain shadows, build-up areas, various water types, different shapes and depth, diverse land cover, and vegetation mixed water are the 
(a) ${ }_{80250 \%}$

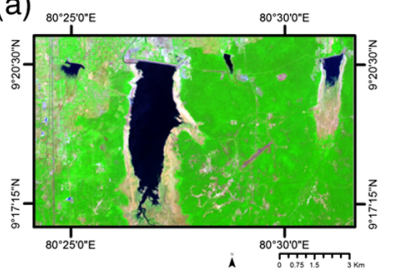

(a') ${ }_{80} 250^{\circ} \mathrm{E}$

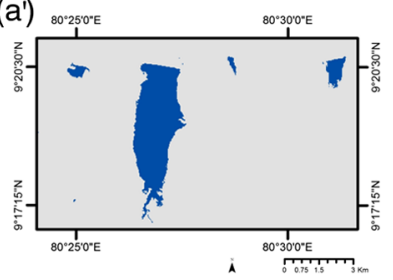

(b)

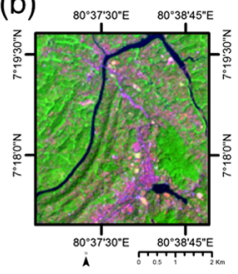

(b')

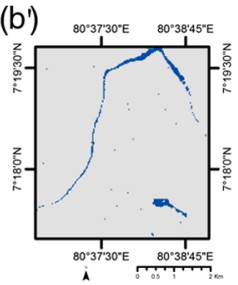

(c)

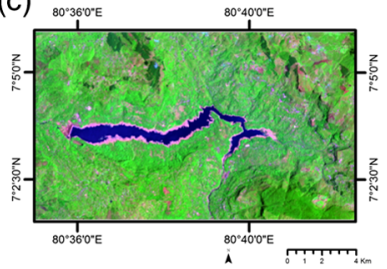

(C') ${ }_{803600 \times}$

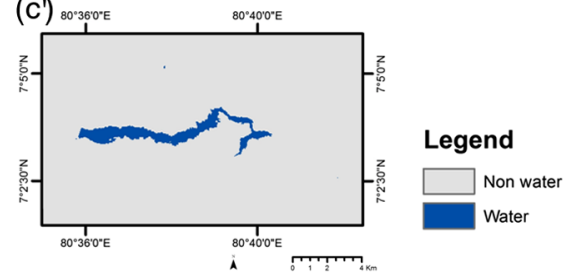

(d)

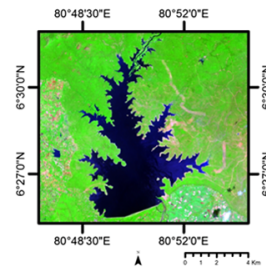

(e)

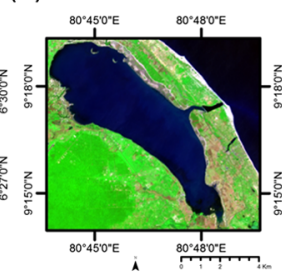

(f)

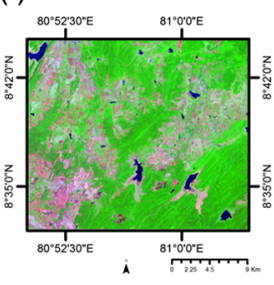

(g) ${ }^{80-5730 \cdot E}{ }^{810000 E}$

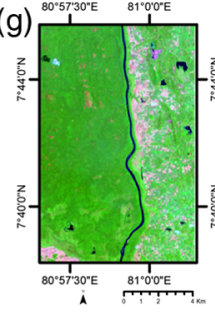

(h)

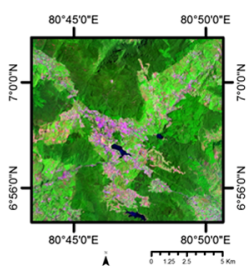

(d')

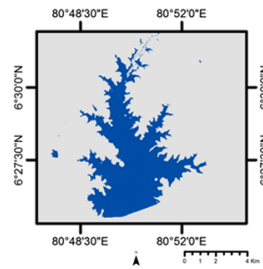

(e')

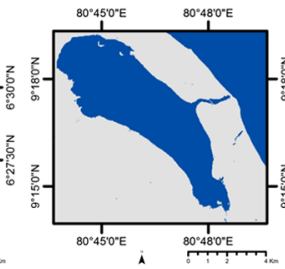

(f')

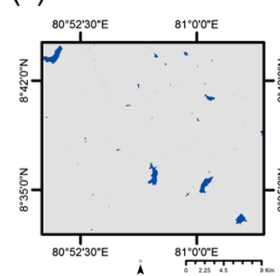

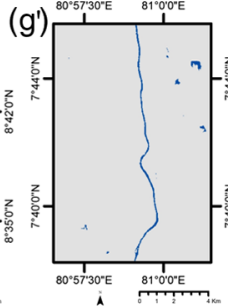

(h')

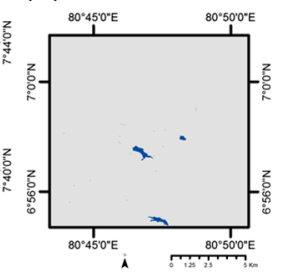

Fig. 4 The extracted water in eight test scenes (a)-(h) are Landsat OLI images with band composite R: band7; G: band5; B: band2 with the results of LVQ.

major influence factors in these test scenes. A simple visual investigation shows that this method succeeded in enhancing variation between water and nonwater in all water environments under confused surroundings and concurrently suppressing low reflectance surfaces in both fresh and coastal waters.

Quantitative assessment of water extraction is done by calculating producer's accuracy, user's accuracy, overall accuracy, and kappa coefficient by constructing error matrices. The results are summarized in Table 2. The overall accuracy and kappa coefficient ranges from $97.80 \%$ to $99.69 \%$ and 0.9559 to 0.9938 , respectively. Similarly, the producer's accuracy and user's accuracy are varied between 0.9717 and 0.9998 and 0.9783 and 0.9945 , respectively. Overall, the results indicate the robustness and higher accurateness of the proposed method under diverse water environmental conditions.

\subsection{Performance Comparison with Other Methods}

The results of LVQ were compared with the results of the five most widely used supervised and unsupervised water detection algorithms: SVM, KNN, DA, combination of modified normalized difference water index and modified fuzzy clustering method (MMFCM), and K-means. SVM ${ }^{41}$ is a hyperplane-based classification technique, $\mathrm{KNN}^{42}$ algorithm is using KNNs for classification, and $\mathrm{DA}^{43}$ is a multidimensional distance parametric classification technique. MMFCM, which is developed by combining modified fuzzy clustering method algorithm, modified normalized difference water index (MNDWI), ${ }^{9}$ and K-means, ${ }^{44}$ which is a popular unsupervised clustering algorithm. All algorithms were implemented in MATLAB R2014b using seven 
Table 2 Accuracy measures of LVQ model in eight test scenes.

\begin{tabular}{lcccc}
\hline \hline $\begin{array}{l}\text { Test } \\
\text { scene }\end{array}$ & $\begin{array}{c}\text { Producer's } \\
\text { accuracy }\end{array}$ & $\begin{array}{c}\text { User's } \\
\text { accuracy }\end{array}$ & $\begin{array}{c}\text { Overall } \\
\text { accuracy (\%) }\end{array}$ & $\begin{array}{c}\text { Kappa } \\
\text { coefficient }\end{array}$ \\
\hline $\mathrm{a}$ & 0.9995 & 0.9926 & 99.61 & 0.9922 \\
$\mathrm{~b}$ & 0.9717 & 0.9841 & 97.80 & 0.9559 \\
$\mathrm{c}$ & 0.9846 & 0.9783 & 98.14 & 0.9627 \\
$\mathrm{~d}$ & 0.9838 & 0.9908 & 98.73 & 0.9745 \\
$\mathrm{e}$ & 0.9998 & 0.9940 & 99.69 & 0.9938 \\
$\mathrm{f}$ & 0.9790 & 0.9945 & 98.68 & 0.9736 \\
$\mathrm{~g}$ & 0.9826 & 0.9842 & 98.34 & 0.9667 \\
$\mathrm{~h}$ & 0.9838 & 0.9815 & 98.27 & 0.9654 \\
\hline \hline
\end{tabular}

OLI bands and NDVI. Water extraction was carried out independently for another 10 various noise-induced scenes using LVQ, SVM (type: CSVC, kernel: radial basis), KNN (NN: 9), DA (type: linear), MMFCM, and K-means clustering, and the result is visually inspected. Furthermore, the ability to suppress shadows, the potential to detect small waters, shadowed water, and the capability of extracting boundaries in more detail without the influence of surroundings and noises are expressed from those test images (Fig. 5). The differences in performance among them are marked as red squares.

Water extraction result in Fig. 5(a) shows that cloud shadow over the paddy fields can be suppressed by LVQ, KNN, and DA [Figs. 5(a1), 5(a3), and 5(a4)], while SVM, MMFCM, and K-means misclassify a portion of cloud shadow as water. In the presence of a large amount of cloud over water and build-up area [Fig. 5(b)], MMFCM showed confusion with cloud shadow and cloud as water; meanwhile, SVM, DA, and K-means clustering method extracted portions of the shadow as water. However, LVQ and KNN were successful in discrimination and accurate identification of shadowed water boundary in the build-up area. In case of terrain shadow, all algorithms exposed similarity in the performance as LVQ [Fig. 5(c)] in contrast to the K-means clustering, which overclassifies terrain shadows as water.

LVQ has the potential to ascertain a very shallow and thin sandy river, which has a similar ground condition in the underwater and adjacent nonwater areas that were partially detected by KNN, DA, MMFCM, and K-means clustering [Fig. 5(d)]. SVM achieved almost the same performance as the LVQ. Water assorted with vegetation, including emergent, floating-leaved, submerged, and free-floating macrophytes [Fig. 5(e)] can be precisely extracted by LVQ, KNN, and DA, while other methods cause commission error. Furthermore, it can clearly be seen that LVQ performed better when identifying detailed water in cases of paddy fields, sand beds, and vegetation mixed river boundaries [Fig. 5(f)] than all other methods, while several portions of sand beds and paddy fields are missed by SVM, KNN, DA, MMFCM, K-means, and some are overclassified by SVM, MMFCM, and K-means.

Seawater, natural and manmade coastal details [Fig. 5(g)] were properly extracted by LVQ, SVM, and KNN with almost similar performance, while KNN misses few areas [Fig. 5(g3)]. Other methods were underclassifying several saltpans and overclassifying manmade structures as water. Result of tank bunds and a narrow line of trees located within a reservoir [Fig. 5(h)] demonstrated that the performance of LVQ, SVM, KNN, and DA was the same; however, KNN and DA were missed some portions of small water bodies and area along tank bunds [Figs. 5(h3) and 5(h4)]. Inner details of the reservoir were not able to be mapped by both MMFCM and K-means [Figs. 5(h5) and 5(h6)]. In addition, relatively greater robustness of LVQ is indicated by the extracted single, double, and eight-pixel sized artificial water bodies [Fig. 5(i1)]. However, the single-pixel feature was undetected by KNN and MMFCM; meanwhile, the KNN and K-means method only partially detected the eight-pixel water body. However, SVM and DA have commissioned few more pixels as water [Fig. 5(i2) and 5(i4)]. These all performances 
(a)

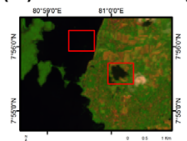

(b)

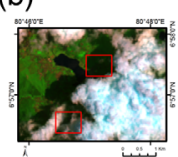

(c)

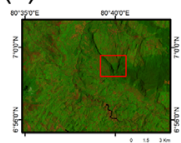

(d)

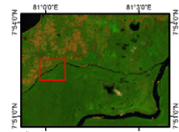

(e)

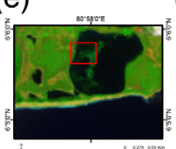

(f)

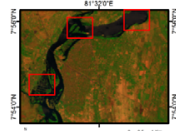

(g)

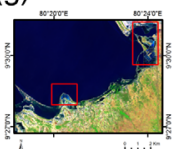

(h)

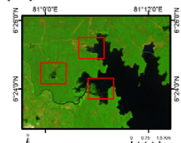

(i)
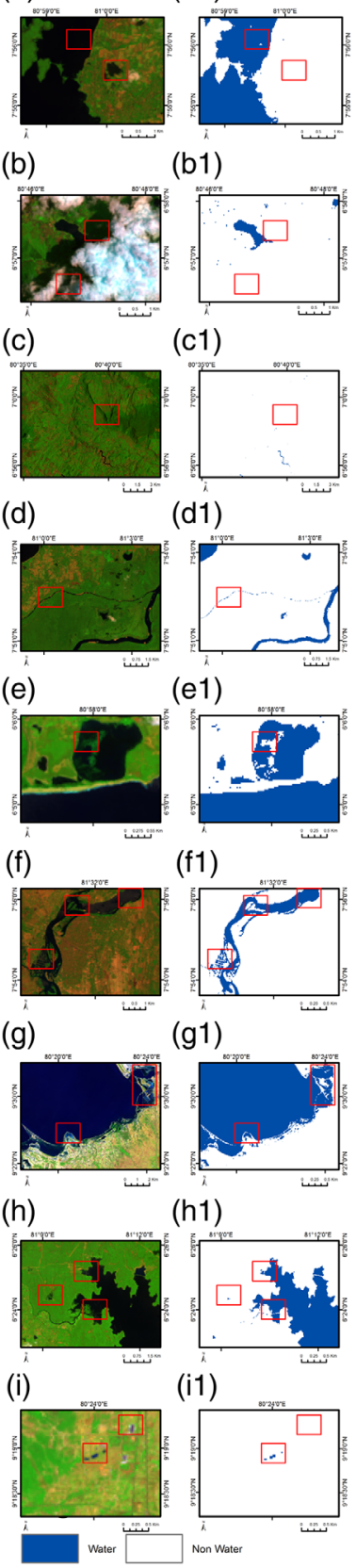

(b1)

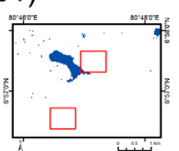

(c1)

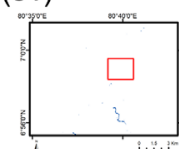

(d1)

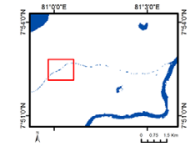

(e1)

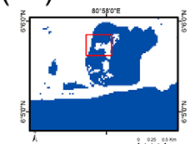

(f1)

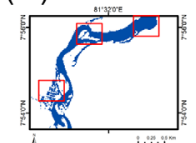

(g1)

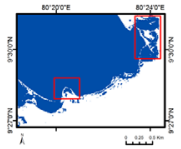

(h1)

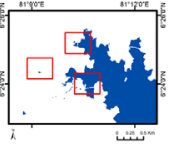

(i1) (a2)

(i2)

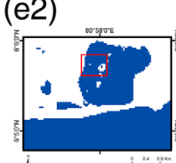

(f2)

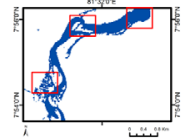

(g2)

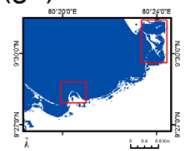

(h2)
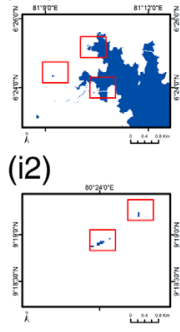

(a3)

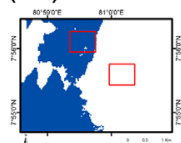

(b3)

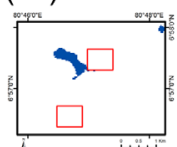

(c3)

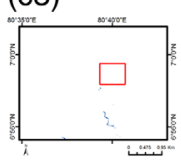

(d3)

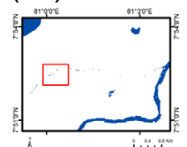

(e3)

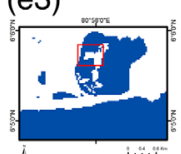

(f3)

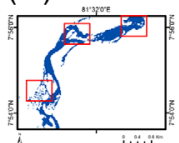

(g3)

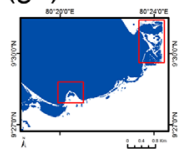

(h3)

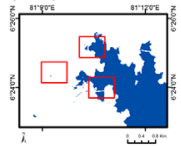

(i3)

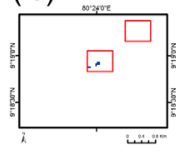

(a4)

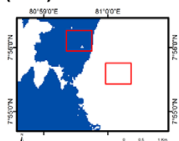

(b4)

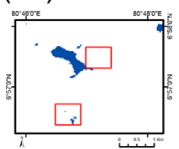

(c4)

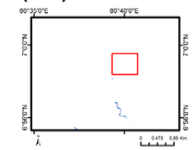

(d4)

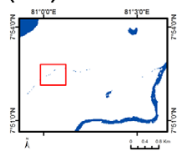

(e4)

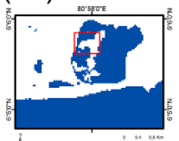

(f4)

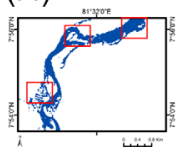

(g4)

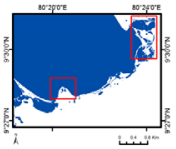

(h4)

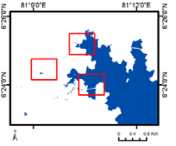

(i4)

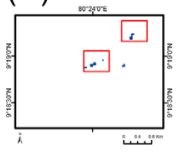

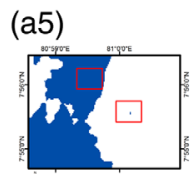

(a6)

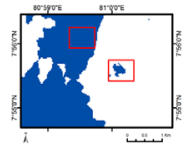

(b6)

(b5)

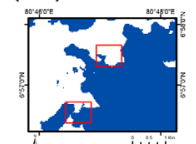

(c5)

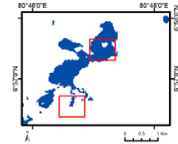

(c6)

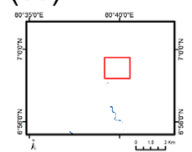

(d5)

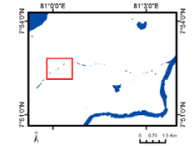

(e5)

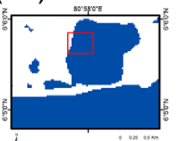

(d6)

(f5)

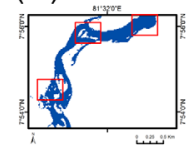

(g5)

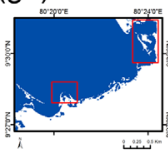

(h5)

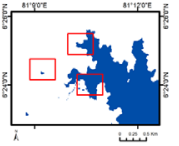

(i5)

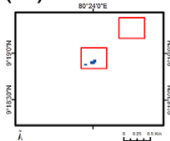

(e6)
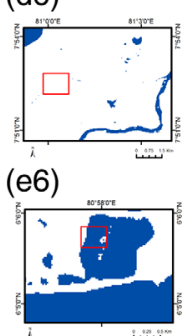

(f6)

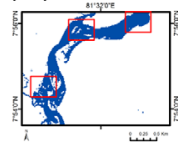

(g6)

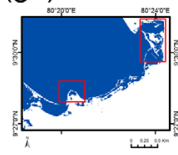

(h6)

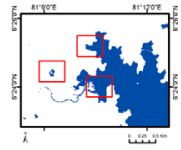

(i6)

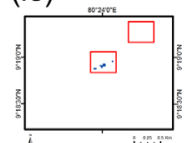

Fig. 5 Performance comparison of surface water extraction by (a1)-(f1) LVQ, (a2)-(f2) SVM, (a3)-(f3) KNN, (a4)-(f4) DA, (a5)-(f5) MMFCM, and (a6)-(f6) K-means under different confusing environments. Difference in performances marked as red squares. Performance comparison of surface water extraction by (a1)-(f1) LVQ, (a2)-(f2) MMFCM, and (a3)-(f3) K-means under different conditions. Variation in performances marked as red squares.

can be concluded that LVQ has depicted a higher capacity to detect different types of water distribution in detail with superior accuracy and stability among the six methods under various confusing states.

\section{Discussion}

This study also revealed that the superior big data generalization ability of LVQ by learning from lesser number of training samples with acceptable accuracy. The number and types of training 
samples were reduced as much as possible to be small using a trial-and-error technique. Similar to these results, studies have already shown empirical evidence for the excellent generalization ability of $\mathrm{LVQ}^{45}$ and $\mathrm{ANN}$ methods in water extractions ${ }^{2}$ using very few training samples. Further, results revealed that LVQ can correctly segment rivers, streams, ponds, reservoirs, ocean water, and saltpans even if the training samples were not provided for those all water types. These may be due to the dynamic learning nature and segmenting characteristics of $\mathrm{LVQ}^{23}$ or the adequateness of the input data in the representation of precise water ${ }^{18}$ or spectral variation of the dataset. Previously, studies stated that the performance of the LVQ-based water detection largely depends on the selection of training samples. ${ }^{4,32}$ This task was highly challenging due to the significant variations in the characteristics of water and nonwater across Sri Lanka. These challenges were accomplished by careful manual selection of samples as much as possible to represent the variation. Perfection in the selection of training samples and parameters may further improve the accuracy. Furthermore, the accuracy of the per-pixel labeling of training samples is a crucial factor that affects the accuracy of the predictions of each pixel during segmentation. In concern of high precision, only well-known water pixels were labeled as water. As a consequence of this process, our model consisted of higher accuracy with minimal omission and commission errors.

An earlier study had reported that scarcity of water training samples in the scenes results in an adverse effect on the output accuracy of LVQ. ${ }^{4}$ This study used training samples from all OLI imageries as same class pixels almost showed similar spectral response in all bands ${ }^{46}$ with several considerations: (1) to achieve accurate water extraction in the scenes that lack water pixels and (2) to speed up the prediction process by reducing human interaction in sample selection and the training process in each scene. Moreover, scenes with small, narrow, various depth and diverse conditional water bodies are much more challenging ${ }^{33,40}$ in contrast to the scenes with larger water features or the majority of water. Thus, the test scenes were selected with various sized water and cover areas range between $0.44 \%$ and $42 \%$ to evaluate the consistency. This model showed good effectiveness to segment water without any limitations in extents [single pixel in Fig. 5(i)], shapes, depths [about $87 \mathrm{~m}$ in Fig. 4(c)], and quantities of water pixels by generating a prime result, as illustrated by Fig. 6 . However, the method leads to false-positive pixels in urban areas (test site b).

In this study, evaluations were conducted through four OLI imageries and therefore, can be considered as a general representative of the country's water features. However, clear sky and availability of very high-resolutional Sentinel-2 images on the same or close proximity date to evaluate accuracy are the main reasons for limited scenes. Manual interference is required in the training processes for appropriate training samples and training parameters selection and perpixel labeling. In addition, the requirement of time for training the LVQ and $\mathrm{ANN}^{2,32}$ is quite considerable. This is due to the training behavior of the neural network and is proportional to the dimensionality of input data. However, it is noted that the time required for the simulation of all images is minimal when the LVQ has been trained.

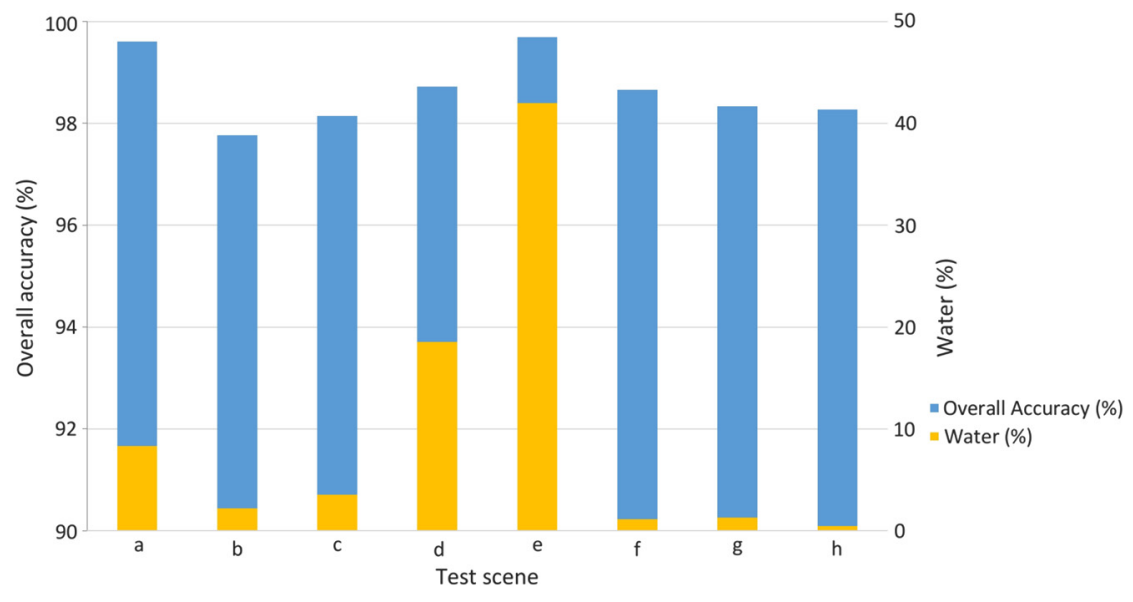

Fig. 6 Comparison of water pixels with accuracy. 


\subsection{Optimum Input Selection}

Surface water extraction is generally impacted by the presence of various forms of environmental noises, surroundings, extent, shape, depth of water, and types of water surfaces. In Sri Lanka, with its tropical and heterogeneity space regions, it is more challenging, because it is always covered by plentiful clouds, cloud shadows, terrain shadows, shadowed water, and higher aquatic plant diversity. These factors may contribute to the confusion during the spectral identification process, especially among small and higher density water features, ${ }^{4}$ which showed that learning from few bands may be insufficient for water identification using LVQ, whereas the accuracy that may increase with the number of bands can be used. ${ }^{28,47}$ A recent study showed the OLI band 7 with good performance in the perspective of water identification. ${ }^{48}$ These all made it a necessity to incorporate more elements to represent real water during the process of learning in highly complex areas as our study site. Hence, a combination of seven OLI spectral bands with NDVI distribution ${ }^{49}$ was introduced as input data to increase accuracy.

Selection of the best training input was carried out using a trial-and-error method with a validation dataset by comparing scenes with noises, such as cloud, terrain shadow, and cloud shadow with complex terrain and land covers, which resulted in the highest overall accuracy and kappa coefficient. Seven OLI bands and NDVI were tested with a few more water indices, and the outcomes are shown in Fig. 7. All produced visually similar results, whereas cloud, cloud shadows, and terrain shadows are significantly contributed to the false positive in Figs. 7(a2) and 7(b2). Results showed that the combination of OLI bands and NDVI index is the ideal and delivers remarkable results [Figs. 6(a1) and 6(a2)] since the accuracy cannot be increased by further adding indices, such as NDWI ${ }^{2}$ and MNDWI.

\subsection{Potential Global Applicability}

The proposed model relies only on Landsat 8 OLI bands, without demanding auxiliary data or prior statistical knowledge on data distribution. Limited training samples showed the ability to detect a number of water type with acceptable accuracy. This model has excellent capacity and flexibility in the architecture to learn and segment in an automated mode at a large scale. Therefore, this model can be implemented to monitor global water by training more spectrally and geometrically varied water samples across the world, from available global water datasets: GLC2000,$^{50}$ Global Land Survey datasets ${ }^{51}$ and Global Land Cover Facility together with perpixel labels, ${ }^{52}$ without any preprocessing ${ }^{20}$ in automated manner.

Learning directly from pixels, without considering data distribution makes the possibility to combine multisource data. Previous studies showed that ANN is insensitive to the input multispectral sensor ${ }^{2,31}$ as well. This method can be easily adapted to other multispectral sensors, such as Quickbird ${ }^{31}$ and Sentinel 2A/B. Future work will be directed toward the time series from Landsat $4,{ }^{4}$ Landsat 5 , and Landsat 7 with available open-source tools. ${ }^{7}$

(a)

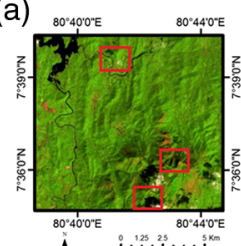

(b)

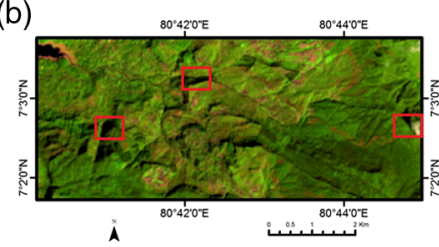

(a1)

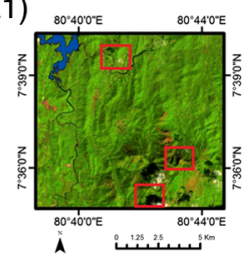

(b1)

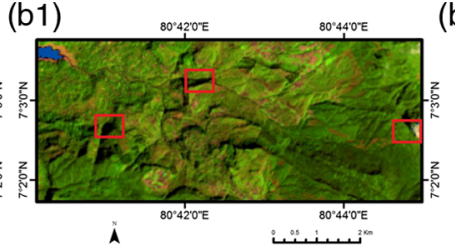

(a2)

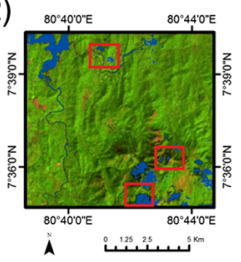

(b2)

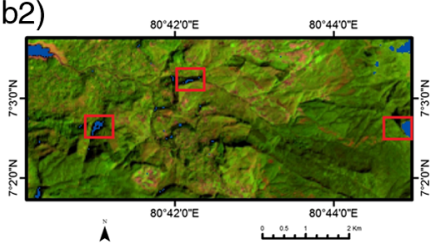

Fig. 7 Performance comparison of surface water extraction by various input layer combinations: (a1), (b1) OLI bands 1 to 7 and NDVI; (a2), (b2) OLI bands 1 to 7, NDVI, NDWI, and MNDWI. Commission errors are marked as red squares. 


\section{Conclusion}

Surface water mapping is getting more attention due to the growing concern about freshwater availability and water-related issues. This study proposes an LVQ neural network model for water extraction using Landsat 8 OLI images, where the heuristic learning process is intrinsically related to the spectral signature, shape, textural information, and spatial dependence. The performance of this model is evaluated under different backgrounds and compared with five commonly used machine learning algorithms (SVM, KNN, DA, MMFCM, and K-means).

The results of the study can be summarized as follows:

(a) Performance of LVQ illustrates that it can accurately identify water with various water types (freshwater and seawater), extents (very narrow and small), shapes, depths, and detailed water boundary that can be extracted. The overall accuracy in eight test sites ranges from $97.8 \%$ to $99.7 \%$ and the kappa coefficients between 0.96 and 0.99 .

(b) According to the visual comparison, LVQ depicted a better performance under the complicated water environments than under the other five algorithms. Results show that it could effectively identify water by suppressing dark surfaces and environmental noises, such as cloud shadow, terrain shadow, build-up, cloud, and floating vegetation. In addition, it is able to precisely detect water under the shadow.

(c) This study also found that the resulted accuracy is acceptable for all types of water bodies and water-scarce scenes, even if training samples are limited in quantity and water types. Accuracy may be further improved with perfection in the training samples and parameters.

(d) The addition of the NDVI improved the consistency of LVQ in the complicated scenes by overcoming noises. Adding more indices did not show improvement in the performance.

Our results demonstrate that LVQ could be very useful for the accurate automated classification of surface water. Further applications remain to be explored, and we suggest that multisensors and universal water samples may be used to understand the global water dynamics over time and to forecast future trends.

\section{Acknowledgments}

The authors are grateful to the U.S. Geological Survey (USGS) and European Space Agency (ESA) for providing the Landsat OLI and Sentinel data. This work was supported by the National Natural Science Foundation of China (Grant Nos. 41701402, 91638201, and 41471308) and the Strategic Priority Research Program of the Chinese Academy of Sciences (Grant No. XDA19080304). The study was also sponsored by the CAS-TWAS President's PhD Fellowship Program awarded to Deepakrishna Somasundaram. No potential conflict of interest was reported by the authors.

\section{References}

1. C. Huang et al., "Detecting, extracting, and monitoring surface water from space using optical sensors: a review," Rev. Geophys. 56(2), 333-360 (2018).

2. A. Paul, D. Tripathi, and D. Dutta, "Application and comparison of advanced supervised classifiers in extraction of water bodies from remote sensing images," Sustain. Water Resour. Manag. 4(4), 905-919 (2017).

3. J. Senthilnath et al., "An approach to multi-temporal MODIS image analysis using image classification and segmentation," Adv. Space Res. 50(9), 1274-1287 (2012).

4. K. Wang and Y. Zhu, "Recognition of water bodies from remotely sensed imagery by using neural network," CSE873 Computer Vision, University of Nebraska-Lincoln (2010).

5. P. Rao et al., "Dynamic change analysis of surface water in the Yangtze River basin based on MODIS products," Remote Sens. 10(7), 1025 (2018).

6. I. Olthof, "Mapping seasonal inundation frequency (1985-2016) along the St-John River, New Brunswick, Canada using the Landsat Archive," Remote Sens. 9(2), 143 (2017). 
Somasundaram et al.: Learning vector quantization neural network for surface water extraction...

7. K. Rithin Paul Reddy et al., Evaluation of Water Body Extraction from Satellite Images Using Open-Source Tools, pp. 129-140, Springer, Singapore (2020).

8. K. Huang et al., "Spectral-spatial hyperspectral image classification based on KNN," Sens. Imaging 17(1), 1 (2016).

9. Y. Yang et al., "Landsat 8 OLI image based terrestrial water extraction from heterogeneous backgrounds using a reflectance homogenization approach," Remote Sens. Environ. 171, 14-32 (2015).

10. K. R. Ahmed and S. Akter, "Analysis of landcover change in southwest Bengal delta due to floods by NDVI, NDWI and K-means cluster with Landsat multi-spectral surface reflectance satellite data," Remote Sens. Appl. Soc. Environ. 8, 168-181 (2017).

11. D. Nova and P. A. Estévez, "A review of learning vector quantization classifiers," Neural Comput. Appl. 25(3-4), 511-524 (2014).

12. J. Brinkrolf, C. Göpfert, and B. Hammer, "Differential privacy for learning vector quantization," Neurocomputing 342, 125-136 (2019).

13. M. Moradi, A. Chaibakhsh, and A. Ramezani, "An intelligent hybrid technique for fault detection and condition monitoring of a thermal power plant," Appl. Math. Model. 60, 34-47 (2018).

14. P. M. Shakeel et al., "Maintaining security and privacy in health care system using learning based deep-Q-networks," J. Med. Syst. 42(10), 186 (2018).

15. A. Zhang et al., "Classification of post-earthquake high resolution image using adaptive dynamic region merging and gravitational self-organizing maps," in Geological Disaster Monitoring Based on Sensor Networks, T. S. Durrani, W. Wang, and S. M. Forbes, Eds., Springer, Singapore (2019).

16. J. Schmidhuber, "Deep learning in neural networks: an overview," Neural Networks 61, 85-117 (2015).

17. W. Liu et al., "A survey of deep neural network architectures and their applications," Neurocomputing 234(November 2016), 11-26 (2017).

18. Z. Zhang, Multivariate Time Series Analysis in Climate and Environmental Research, Springer International Publishing, Cham (2018).

19. K. Rokni et al., "A new approach for surface water change detection: integration of pixel level image fusion and image classification techniques," Int. J. Appl. Earth Obs. Geoinf. 34(1), 226-234 (2015).

20. F. Isikdogan, A. C. Bovik, and P. Passalacqua, "Surface water mapping by deep learning," IEEE J. Sel. Top. Appl. Earth Obs. Remote Sens. 10(11), 4909-4918 (2017).

21. Z. Zeng, J. Liu, and H. H. G. Savenije, "A simple approach to assess water scarcity integrating water quantity and quality," Ecol. Indic. 34, 441-449 (2013).

22. J. Amezcua and P. Melin, "A new fuzzy learning vector quantization method for classification problems based on a granular approach," Granul. Comput. 4(2), 197-209 (2019).

23. M. Biehl, A. Ghosh, and B. Hammer, "Dynamics and generalization ability of LVQ algorithms," J. Mach. Learn. Res. 8, 323-360 (2007).

24. M. Biehl, B. Hammer, and T. Villmann, "Prototype-based models in machine learning," Wiley Interdiscip. Rev. Cogn. Sci. 7(2), 92-111 (2016).

25. A. Ghosh, M. Biehl, and B. Hammer, "Performance analysis of LVQ algorithms: a statistical physics approach," Neural Networks 19(6-7), 817-829 (2006).

26. S. Bashyal and G. K. Venayagamoorthy, "Recognition of facial expressions using Gabor wavelets and learning vector quantization," Eng. Appl. Artif. Intell. 21(7), 1056-1064 (2008).

27. A. M. Filippi and J. R. Jensen, "Fuzzy learning vector quantization for hyperspectral coastal vegetation classification," Remote Sens. Environ. 100(4), 512-530 (2006).

28. H. Bagan et al., "Land-cover classification using ASTER multi-band combinations based on wavelet fusion and SOM neural network," Photogramm. Eng. Remote Sens. 74(3), 333-342 (2008).

29. Y. Chen, N. M. Nasrabadi, and T. D. Tran, "Discriminative dictionary design using LVQ for hyperspectral image classification," in 4th Workshop Hyperspectral Image and Signal Process. (WHISPERS), IEEE, pp. 1-4 (2012). 
30. Z. Wang, N. M. Nasrabadi, and T. S. Huang, "Spatial-spectral classification of hyperspectral images using discriminative dictionary designed by learning vector quantization," IEEE Trans. Geosci. Remote Sens. 52(8), 4808-4822 (2014).

31. S. Deepakrishna, S. Ediriweera, and A. A. J. K. Gunatilake, "Automatic feature extraction from satellite images using LVQ neural network," in 37th Asian Conf. Remote Sens. (2016).

32. F. S. Erbek, C. Özkan, and M. Taberner, "Comparison of maximum likelihood classification method with supervised artificial neural network algorithms for land use activities," Int. J. Remote Sens. 25(9), 1733-1748 (2004).

33. A. Fisher, N. Flood, and T. Danaher, "Comparing Landsat water index methods for automated water classification in eastern Australia," Remote Sens. Environ. 175, 167-182 (2016).

34. S. H. S. Senarathne et al., "Feasibility of using problematic aquatic weeds in productive manner by generating vermicompost in coconut triangle area of Sri Lanka," Int. J. Coconut Res. Dev. 33(1), 16-30 (2017).

35. USGS, "EarthExplorer - home," EarthExplorer, 2018, https://earthexplorer.usgs.gov/ (accessed 12 March 2019).

36. ESA, "Sentinel online - ESA," 2019, https://sentinel.esa.int/web/sentinel/home (accessed 24 January 2019).

37. N. E. Young et al., "A survival guide to Landsat preprocessing," Ecology 98(4), 920-932 (2017).

38. L. Feng et al., "Exploring the potential of Rayleigh-corrected reflectance in coastal and inland water applications: a simple aerosol correction method and its merits," ISPRS J. Photogramm. Remote Sens. 146, 52-64 (2018).

39. B. Biswal et al., "Nonstationary power signal time series data classification using LVQ classifier," Appl. Soft Comput. 18, 158-166 (2014).

40. T. D. Acharya, A. Subedi, and D. H. Lee, "Evaluation of water indices for surface water extraction in a Landsat 8 scene of Nepal," Sensors (Switzerland) 18(8), 2580 (2018).

41. V. Vapnik, "The support vector method of function estimation," in Nonlinear Modeling, J. A. K. Suykens and J. Vandewalle, Eds., pp. 55-85, Springer US, Boston, Massachusetts (1998).

42. T. Cover and P. Hart, "Nearest neighbor pattern classification," IEEE Trans. Inf. Theory 13(1), 21-27 (1967).

43. S. J. Press and S. Wilson, "Choosing between logistic regression and discriminant analysis," J. Am. Stat. Assoc. 73(364), 699-705 (1978).

44. J. MacQueen, "Some methods for classification and analysis of multivariate observations," in Proc. Fifth Berkeley Symp. Math. Stat. and Probab., Volume 1: Statistics, University of California Press, Berkeley, California, pp. 281-297 (1967).

45. J. Liu et al., "Nonwoven uniformity identification using wavelet texture analysis and LVQ neural network," Expert Syst. Appl. 37(3), 2241-2246 (2010).

46. K. Makantasis et al., "Deep supervised learning for hyperspectral data classification through convolutional neural networks," in IEEE Int. Geosci. and Remote Sens. Symp. (IGARSS), IEEE, pp. 4959-4962 (2015).

47. W. Jiang et al., "Multilayer perceptron neural network for surface water extraction in Landsat 8 OLI satellite images," Remote Sens. 10(5), 755 (2018).

48. H. Xie et al., "Evaluation of Landsat 8 OLI imagery for unsupervised inland water extraction," Int. J. Remote Sens. 37(8), 1826-1844 (2016).

49. X. Song, Z. Liu, and Y. Zhao, "Cloud detection and analysis of MODIS image," in Proc. IEEE Int. Geosci. and Remote Sens. Symp., IGARSS '04, IEEE, vol. 4, pp. 2764-2767 (2004).

50. E. Bartholomé and A. S. Belward, "GLC2000: a new approach to global land cover mapping from Earth observation data," Int. J. Remote Sens. 26(9), 1959-1977 (2005).

51. G. Gutman et al., "Assessment of the NASA-USGS Global Land Survey (GLS) datasets," Remote Sens. Environ. 134, 249-265 (2013).

52. M. Feng et al., "A global, high-resolution (30-m) inland water body dataset for 2000: first results of a topographic-spectral classification algorithm," Int. J. Digital Earth 9(2), 113-133 (2016). 
Deepakrishna Somasundaram received her MSc degree in geographical information system and remote sensing from the University of Peradeniya, Sri Lanka, in 2016. She is currently a PhD student in the Institute of Remote Sensing and Digital Earth, Chinese Academy of Sciences, China. Her research interests include surveying and mapping, machine learning and their applications in remote sensing, and watercolor remote sensing.

Fangfang Zhang is an associate professor at the Institute of Remote Sensing \& Digital Earth, Chinese Academy of Sciences. He received his $\mathrm{PhD}$ in cartography and geographic information system from East China Normal University, Shanghai, China, in 2014. His current research interest is watercolor remote sensing, mainly including the development of estimation models for water quality parameters and software for operational monitoring water quality.

Shenglei Wang received a BS degree in geographic information system from Northeast Forestry University, an MS degree in photogrammetry and remote sensing from Central South University, Changsha, China, and a PhD in cartography and geographic information system from the University of Chinese Academy of Sciences in 2018. She is currently a postdoc research fellow at Peking University, Beijing, China. Her research interests include water optical remote sensing, bio-optical properties and radiative transfer process in optically-complex waters, and spatiotemporal change analysis of watercolor and the responses to climate change.

Huping Ye received his $\mathrm{PhD}$ in cartography and geographic information system from the Institute of Remote Sensing and Digital Earth, Chinese Academy of Sciences, Beijing, China, in 2017. He is an assistant professor at the Institute of Geographic Sciences and Natural Resources Research, Chinese Academy of Sciences, Beijing, China. His current research interests include unmanned aerial vehicle (UAV) remote sensing data process, watercolor remote sensing and water optics, mainly including remote sensing inversion of water quality parameters, inherent optical properties measurement, and inversion.

Zongke Zhang is a professor of the Institute of Remote Sensing and Digital Earth (RADI), Chinese Academy of Sciences (CAS). His research interests are remote sensing and GIS technology in resources and ecological environment monitoring, natural disasters and large-scale engineering environmental assessment, information system development and application.

Bing Zhang is a full professor of the Institute of Remote Sensing and Digital Earth (RADI), Chinese Academy of Sciences (CAS). His research interests include the development of mathematical and physical models and image processing software for the analysis of remote sensing data in many different areas. He has authored more than 300 publications, including more than 200 journal papers. He currently serves as the associate editor for the IEEE Journal of Selected Topics in Applied Earth Observations and Remote Sensing. He has been the guest editor of special issues of IEEE JSTARS, IEEE GRSL, Proceedings of IEEE, Pattern Recognition Letters, etc. He also has been serving as the technical committee member of IEEE Workshop on Hyperspectral Image and Signal Processing (IEEE WHISPERS) since 2011 and as the president of hyperspectral remote sensing committee of China National Committee of International Society for Digital Earth since 2012, and as the standing director of Chinese Society of Space Research (CSSR) since 2016. 